\title{
Treatment for Rheumatoid Arthritis
}

3 Wen Zheng ${ }^{\mathrm{a}, \uparrow}$, Xianghong $\mathrm{Lu}^{\mathrm{b}, \dagger}$, Zhirong Fu ${ }^{\mathrm{a}}$, Lin Zhang ${ }^{\mathrm{a}}$, Ximin $\mathrm{Li}^{\mathrm{a}}$, Xiaobao Xu ${ }^{\mathrm{a}}$, Yina Ren ${ }^{\mathrm{a}}$,

4 Yongzhuang $\mathrm{Lu}^{\mathrm{a}}$, Hongwei $\mathrm{Fu}^{\mathrm{a}}$, and Jingkui Tian ${ }^{* \mathrm{a}, \mathrm{c}}$

5 College of Biomedical Engineering and Instrument Science, Zhejiang University, Hangzhou,

6 Zhejiang, 310027, China

$7 \quad$ b Lishui People's Hospital, Lishui, Zhejiang, 323000, China

$8{ }^{\mathrm{c}}$ Education Ministry Key Laboratory for Biomedical Engineering, Zhejiang University,

9 Hangzhou, Zhejiang, 310027, China

10 These authors have contributed equally to this work.

$11 *$ Corresponding author: Jing-kui Tian, Zhouyi Qing Building, Room 108, College of

Biomedical Engineering and Instrument Science, Zhejiang University, Hangzhou, Zhejiang,

310027, China. E-mail: tjk@ zju.edu.cn. Tel: +86-057187951301; Fax: +86 057187951091.

Abbreviations: 2D-DIGE, two-dimensional DIGE; CFA, complete Freund's adjuvant; CIA,

Collagen-induced arthritis; CII, bovine type II collagen; CMC-Na, sodium

carboxymethylcellulose; DEX, dexamethasone; H\&E, haematoxylin and eosin; NMR, nuclear magnetic resonance; OA, Osteoarthritis; RA, Rheumatoid arthritis; SpA, Spondyloarthropathy; TCMs, traditional Chinese medicines; TWH, Tripterygium wilfordii Hook; RA, Rheumatoid arthritis; CIA, Collagen-induced arthritis; SpA, Spondyloarthropathy; OA, Osteoarthritis

Key words: Achyranthes aspera, Saponins, Rheumatoid arthritis, two-dimensional DIGE 


\section{ABSTRACT}

Rheumatoid arthritis (RA) is a systemic autoimmune disease whose main symptom is a heightened inflammatory response in synovial tissues. To verify the anti-arthritic activities of Achyranthes aspera and its possible therapy-related factors on the pathogenesis of RA, the saponins in A. aspera root were isolated and identified to treat the collagen-induced arthritis (CIA) rats. Phytochemical analysis isolated and identified methyl caffeate, 25-S-inokosterone, 25-S-inokosterone $\quad \beta$-D-Glucopyranosyl $3-(\mathrm{O}-\beta$-D-glucopyranosyloxy)-oleanolate, and
$\beta$-D-Glucopyranosyl
3-(O- $\beta$-D-glactopyranosyl
$(1 \rightarrow 2)(\mathrm{O}-\beta-\mathrm{D}-$ glucopyranosyloxy)-oleanolate as main compounds in root of Achyranthes aspera. Proteomics was performed to determine the differentially expressed proteins in either inflamed or drug-treated synovium of CIA rats. Treatment resulted in dramatically decreased paw swelling, proliferation of inflammatory cells, and bone degradation. Fibrinogen, procollagen, protein disulfide-isomerase A3, and apolipoprotein A-I were all increased in inflamed synovial tissues and were found to decrease when administered drug therapy. Furthermore, Alpha-1-antiproteinase and manganese superoxide dismutase were both increased in drug-treated synovial tissues. The inhibition of RA progression shows that $A$. aspera is a promising candidate for future treatment of human arthritis. Importantly, the total saponins found within A. aspera are the active component. Finally, autoantigens such as fibrinogen and collagen could act as inducers of RA due to their aggravation of inflammation.

Given this, it is possible that the vimentin and PDIA3 could be the candidate biomarkers specific to Achyranthes saponins therapy for rheumatoid arthritis in synovial membrane. 


\section{Introduction}

Rheumatoid arthritis (RA) is an autoimmune disorder that attacks the joints, causing chronic and painful inflammation. There are currently several therapeutic options to treat RA [1]. Interestingly, many traditional Chinese medicines (TCMs) have been shown to have therapeutic effects on ameliorating arthritic symptoms for their immunosuppression, anti-inflammation, and/or antioxidant activity [2]. The She are one of China's ethnic minorities where. They reside in the southern mountain areas, which have a humid climate that induces pain level of RA [3, 4]. During the two thousand years they have spent fighting the disease, the She people have found that a decoction of Achyranthes aspera L. (Amaranthaceae) has robust efficacy for use as an RA therapy, however, the clinical effect has not been proved in report yet.

A. aspera is a traditional medicinal plant used in many Asian countries. The non-alkaloid fraction of the A. aspera leaf possesses a pronounced anti-carcinogenic effect [5]. Moreover, the ecdysterone found in the seeds of $A$. aspera have shown both immunostimulatory and growth promoting properties in the larvae of the common carp Cyprinus carpio [6]. Extracts of A. aspera also possess in vivo wound healing activity [7]. They have also been used as folk remedies for reducing internal heat due to increased metabolism in the disease state as well as antipyretic, snake bite, and wound healing [8-10]. However, despite the wide and varied use of A. aspera extracts, their pharmaceutical and pharmacological mechanisms have yet to be elucidated.

Finally, the ethyl acetate fraction of the crude extract of A. aspera $(200 \mathrm{mg} / \mathrm{mL})$ has shown anti-inflammatory activity in the carrageenan-induced hind paw edema model [11]. It 
1 has also been reported that using ethanol extracts of A. aspera in the same model results in

2 anti-inflammatory and anti-arthritic activities at doses ranging from $100-500 \mathrm{mg} / \mathrm{kg}[12,13]$.

3 There is intense interest in employing proteomics technology to foster a better

4 understanding of disease processes, develop new biomarkers for diagnoses, for use in the

5 early detection of disease, and to accelerate drug development [14]. Recently, this technology

6 has been used in the study of RA biomarkers found in blood and synovial tissue. The

7 proteomes of different synovial tissues between RA, spondyloarthropathy ( $\mathrm{SpA}$ ), and

8 osteoarthritis (OA) were analyzed. Fructose bisphosphate aldolase A and alpha-enolase were

9 candidate biomarkers used to distinguish between $\mathrm{SpA}$ and OA. Calgranulin A myeloid related protein 8 (MRP 8) was also markedly higher in RA and SpA patients when compared to OA patients, with the latter having levels below the limits of detection [15].

Mass spectrometric proteomic and protein biochip analyses of synovial fluid and plasma from patients suffering from RA and OA investigated the inflammation profile behind different rheumatic pathologies. Calgranulin B and MRP 8 were specific to the synovial fluid collected from RA patients $[16,17]$. Serum amyloid A protein was found solely in RA patients' plasma and synovial fluid [16]. A two-step proteomic approach was used to identify biomarkers of disease severity in the synovial fluid and serum of patients with RA. C-reactive protein, calgranulin $\mathrm{A}, \mathrm{B}$, and $\mathrm{C}$ were identified as potential protein marker candidates for prognosis of the erosive form of RA [18]. A proteomics approach was applied to analyze the proteomic patterns of fibroblast-like synovial cells and peripheral blood mononuclear cells in RA patients. Proteins BiP, colligin, $\mathrm{HC}$ gp-39 were characterized as potential autoantigens in 
the inflamed paws of mice with Collagen-induced arthritis (CIA) showed that ferritin light chain and antioxidant protein were increase while lymphoid enhancer binding factor decrease

3 in inflamed paws which provided more detailed understanding of arthritic joints [20]. These

4 results demonstrate the utility of a proteomics approach in understanding a complex disease

5 like arthritis. Importantly, it has further utility in identifying possible molecular markers for future diagnoses.

In this study, active compounds from A. aspera root were isolated and identified. The

8 CIA model was used in rats to clarify the pharmacological activity of these isolated compounds. To further establish potential biomarkers found during pathogenesis and therapy, a comparative proteomics analysis of synovial tissue in normal, vehicle control, and A. aspera therapy groups was performed using two-dimensional DIGE (2D-DIGE).

\section{Materials and Methods}

2.1 Phytochemical Analysis in A. aspera Root

Fresh A. aspera root was identified by Lin Zhang, an associate professor at the College of Biomedical Engineering and Instrument Science at Zhejiang University. The extraction, isolation, and quantification of saponins protocols are described in detail in the Supplemental Method. All NMR spectra were recorded on a Bruker ARX-500 and ARX-125 MHz NMR spectrometer (Bruker, Bellerica, MA, USA) equipped with a $\mathrm{CH}$ dual $5 \varphi$ probe. Samples were dissolved in $0.6 \mathrm{~mL}$ pyridine- $\mathrm{d}_{5}$ and transferred into a 5-mm NMR tube. All chemical shifts are expressed as $\delta(\mathrm{ppm})$ relative to the internal standard TMS $(\delta=\mathrm{ppm})$ and scalar coupling constants are reported in $\mathrm{Hz}$. 
We used standard and specific pathogen-free (SPF)-Wistar male rats obtained from the

Shanghai SLAC Laboratory Animal Co. Ltd. of the Chinese Academy of Sciences (Shanghai, China). All subjects weighed between 180-200 g. The animals were maintained in a temperature-controlled room in a $12 \mathrm{hrs}$ light-dark cycle with free access to both standard rodent chow and water. All animal care and experimental procedures in accordance with the guidelines and regulations set forth by the Care and Use of Laboratory Animals, formulated by the Ministry of Science and Technology of the People's Republic of China. All work was approved by the Ethical Committee on Animal Experiments at University Zhejiang, Zhejiang, China (SYXK/Zhe/2007-0098).

\subsection{Induction of Type II CIA}

The rats were immunized with $100 \mu \mathrm{g}$ bovine type II collagen (CII) (Chondrex, Redmond, WA) in a 1:1 mixture of complete Freund's adjuvant (CFA) to collagen. Subjects received an injection subcutaneously into the base of the tail. A second booster injection was given seven days after the initial injection. The normal group was injected with normal saline at the same volume as experimental subjects.

\subsection{Drug Treatment and Index Measurement}

The CIA rats were randomly divided into 6 groups of 10 subjects per group. Normal group was injected with normal saline and given $0.5 \%$ sodium carboxymethylcellulose (CMC-Na) daily by gavage. Vehicle control group was immunized with CII-CFA and given 0.5\% CMC-Na daily by gavage. Drug treatment groups were immunized with CII-CFA and administered by gavage daily with 75,150 , or $300 \mathrm{mg} / \mathrm{kg}$ achyranthes extract, $40 \mathrm{mg} / \mathrm{kg}$ 
1 Tripterygium wilfordii Hook (TWH), or $0.3 \mathrm{mg} / \mathrm{kg}$ dexamethasone (DEX), respectively. Each

2 group was given continuous dosings at seventh days of the first immunization and lasted for

314 days in a standard volume of $1 \mathrm{~mL} / 100 \mathrm{~g}$. Amount of hind paw swelling was measured during drug treatment. Volume of swelling was determined using a MK-550 volume meter (Muromachi Kikai Co., Tokyo, Japan) before the initial immunization and repeated every two days thereafter. The paw swelling $(\Delta \mathrm{mL})$ was defined as the paw volume after immunization.

\subsection{Histological Staining}

Twenty days after the initial immunization, the hind paws of all rats were radio-graphed using a high frequency X-ray camera (Perlove, PLX160A, Nanjing, China) with $40 \mathrm{kV}$ and 22 mAs. The radiographs were exported by DRConsole (Perlove, PLX160A, Nanjing, China). After examining the X-rays, rats were sacrificed by diethyl ether asphyxiation and three rats of each group were used for histology examimation. Hind paws were fixed in $10 \%$ formalin for $24 \mathrm{hrs}$ and then decalcified with RapidCal-ImmunoTM (BBC Biochemical, Seattle, WA, USA) for 48 hrs. Tissue samples were dehydrated, paraffin embedded, and sectioned on a microtome (Microm HM-325; MICROM, Walldorf, Germany). A 4- $\mu \mathrm{m}$ paraffin section was stained with haematoxylin and eosin (H\&E). The stained slides were viewed using an Olympus BX51 microscope (Tokyo, Japan) and digital high-resolution microphotographs of the samples were captured for further analysis (Olympus DP 10, Olympus, Tokyo, Japan).

\subsection{Protein Preparation and 2D-DIGE}

Synovium were dissected for the preparation of protein extraction. Four biological 
repicates were used to identify the differentially expressed proteins. Equal amounts of normal,

2 vehicle, and achyranthes-treated $(300 \mathrm{mg} / \mathrm{kg})$ tissue $(100 \mathrm{mg})$ were quick-frozen in liquid

3 nitrogen and ground-up into a fine powder using a mortar and pestle. All samples were

4 solubilized with DIGE lysis buffer (9 M urea, 4\% CHAPS, every $100 \mathrm{mg}$ tissue added $200 \mu \mathrm{L}$

5 buffer) for $1 \mathrm{hr}$ at $30{ }^{\circ} \mathrm{C}$ and the resulting solution was centrifuged twice at $15,000 \mathrm{~g}$ for 15

6 min to remove insoluble substance. Total protein levels were determined by a Bradford assay.

7 The $\mathrm{pH}$ of the extracts was then adjusted to 8.0 with a $50 \mathrm{mM} \mathrm{NaOH}$ solution. Each gel was

8 loaded with $50 \mu \mathrm{g}$ of protein that had been labeled with 400 pmol of CyDye ${ }^{\mathrm{TM}}$ DIGE

9 Fluor minimal dyes (Cy3, Cy5, or Cy2, GE Healthcare, Amersham Biosciences, Uppsala,

10 Sweden). Cy2 was used exclusively to label the internal standard and other samples were

11 labeled by Cy3 and Cy5 shown in Supplementary Table S1. Labeled proteins were kept on ice for $30 \mathrm{~min}$ and then quenched with $1 \mu \mathrm{L}$ lysine $(10 \mathrm{mM})$.

The volume of the samples was then adjusted to $450 \mu \mathrm{L}$ with lysis buffer (9 M urea, $4 \%$ CHAPS, 1\% IPG buffer, 1\% DTT) and loaded onto $24 \mathrm{~cm}, 3-10$ NL IPG strips (GE Healthcare). The details of isoelectric focusing and second dimension electrophoresis were described in Supplementary Method. Gels were scanned using a DIGE Imager Scanner (Typhoon 9400, GE Healthcare) at a resolution of $100 \mu \mathrm{m}$. Image analysis was performed and included spot detection, gel overlay, and normalization based on the pooled internal standard. We then quantified using Decyder2D software according to the manufacturer's instructions (v7.0, GE Healthcare). Volumes of the detected spots were normalized by dividing the sum of spot volumes for all detected spots, and then multiplied by 100 to obtain spot percentage (normalized spot volume, vol \%). Differences in spot intensities were statistically analyzed 
using a Student's t-test. Protein spots that had an average of 50\% variation $(P<0.05)$ were considered as differentially expressed and chosen for further analysis.

\subsection{Silver Staining and Protein Identification}

Gels loaded with $200 \mu \mathrm{g}$ of protein from each group were subjected to 2D-DIGE as described above and silver stained as previously reported [21]. Images were scanned with an ImageScanner (GE Healthcare) at a resolution of 300 dpi and at 256-gray level.

The selected gel spots were manually excised, destained, and washed sequentially with $100 \mu \mathrm{L} 25 \mathrm{mM}$ ammonium bicarbonate $\left(\mathrm{NH}_{4} \mathrm{HCO}_{3}\right), 50 \%$ acetonitrile $(\mathrm{ACN})$ in $25 \mathrm{mM}$ ammonium bicarbonate, and 100\% ACN. Gels were then vacuum-dried, rehydrated in digestion buffer $\left(0.02 \mu \mathrm{g} / \mu \mathrm{L}\right.$ trypsin, in $\left.10 \% \mathrm{ACN} / 25 \mathrm{mM} \mathrm{NH}_{4} \mathrm{HCO}_{3}\right)$, and covered with 20 $\mu \mathrm{L} 10 \% \mathrm{ACN} / \mathrm{NH}_{4} \mathrm{HCO}_{3}$. After $16 \mathrm{~h}$ of digestion at $37{ }^{\circ} \mathrm{C}$, the gels were extracted with $50 \mu \mathrm{L}$ $5 \%$ TFA in $67 \%$ ACN. The digested solution and extracts were mixed and freeze-dried.

MALDI-TOF/TOF mass spectrometry was used in protein identification. Briefly, dried digests were dissolved in $5 \mu \mathrm{L} 0.1 \%$ TFA and mixed with the same volume of matrix solution ( $\alpha$-cyano-4-hydroxycinnamic acid saturated with 50\% ACN/1 \% TFA). A $1 \mu \mathrm{L}$ aliquot of this mixture was deposited onto the MALDI target. Subsequent MS analyses were performed on MALDI-TOF/TOF MS system (ABI 5800, Applied Biosystems, Foster city, CA, USA). Peptides were analyzed in positive ion mode. The MS and MS/MS spectra were combined and searched against the NCBInr database using GPS Explorer (v3.6, Applied Biosystems) and MASCOT (v2.3, Matrix Science). The search parameters were as follows: NCBInr database (20130413, 24,553,352 sequences, 8,469,922,479 residues), taxonomy of Rattus (68,315 sequences), trypsin digest with one missing cleavage, fixed modifications of 
1 carbamidomethyl (C), none fixed modifications of Acetyl (Protein N-term), deamidated (NQ),

2 dioxidation (W), and oxidation (M), MS tolerance of $100 \mathrm{ppm}$, MS/MS tolerance of $0.3 \mathrm{Da}$.

3 The protein ID was chosen by the highest score searched in the database and proteins with at

4 least two peptides were satisfied the requirements. Confidence interval of $95 \%(P<0.05)$ was

$5 \quad$ set as the significance threshold for mascot protein score.

$6 \quad 2.8$ Immunoblotting

7 In order to verify changes in arthritis- and anti-inflammatory- associated proteins of 8 interest, we performed immunoblotting with antibodies (BIOSS, Beijing, China) against 9 selected proteins in synovium of hind paws. The method of protein extraction was the same as described above. Three biological repicates were used for immunoblotting. The protocols

11 were described in detail in the Supplemental Method. 2.9 Statistical Analysis

Data were expressed as means \pm standard deviations (SD) and analyzed using SPSS

14 software (version 16.0, Chicago, IL, USA). 2D-DIGE spot abundance was statistically 15 evaluated using Decyder2D software. Differences between experimental groups and vehicle control group or between normal group and vehicle control group were assessed using paired

17 Student's t test. For all tests, $P<0.05$ was considered significant.

19 3. Results

3.1 Identification of Anti-RA Composition in A. aspera

Four main compounds were isolated and structures were identified by nuclear magnetic 
resonance (NMR) spectroscopy (Supplementary Figure S1). The NMR spectral data were described in Supplementary NMR spectral data. ${ }^{13} \mathrm{C}$ NMR spectral data of compound 3 and 4 are provide in a list in Supplementary Table S2.

Compound 1 was obtained as a colorless, needle-shaped crystal (methanol). The NMR spectral data are consistent with CAS database identification of methyl caffeate (CAS No.3843-74-1). Compound 2 was obtained as a white, needle-shaped crystal (methanol). Spectral data are consistent with previous reports as 25-S-inokosterone [22]. Compound 3 was obtained as a white powder (methanol). Spectral data are consistent with previous reports of 25-S-inokosterone $\quad \beta$-D-Glucopyranosyl $\quad 3$-(O- $\beta$-D-glucopyranosyloxy)-oleanolate $\quad[23]$. Compound 4 was obtained as a white powder (methanol). Spectral data are consistent with previous reports as $\beta$-D-Glucopyranosyl 3 -(O- $\beta$-D-glactopyranosyl $\quad(1 \rightarrow 2)(\mathrm{O}-\beta$-Dglucopyranosyloxy)-oleanolate [23].

Two of these main compounds accounted for a large proportion of the saponin content. The standards of $\beta$-D-Glucopyranosyl 3-(O- $\beta$-D- glucopyranosyloxy)-oleanolate (AA-1) and $\beta$-D-Glucopyranosyl $\quad 3-(\mathrm{O}-\beta-\mathrm{D}-\quad$ glactopyranosyl-( $1 \rightarrow 2)-\quad$ (O- $\beta$-D-glucopyranosyloxy)oleanolate (AA-2) were purified in our lab. According to the calculation method of area normalization, the purity of each AA-1 and AA-2 was more than $98 \%$. These two compounds were carefully weighed and dissolved as control samples in the mobile phase to $0.552 \mathrm{mg} / \mathrm{mL}$ and $0.575 \mathrm{mg} / \mathrm{mL}$, respectively. HPLC chromatogram of total saponins (Black), AA-1 (Green), and AA-2 (Blue) are shown in Supplementary Figure S2. AA-1 and AA-2 were measured in four separate total saponin extracts. The average contents were $17.067 \%$ (AA-1) and $17.50 \%$ (AA-2) (Supplementary Table S3). 
CIA was induced with primary and booster injections of CII/CFA at an interval of 7 days.

3 Experimental groups began receiving treatment (e.g. Achyranthes) beginning on day 8.

4 Arthritis symptoms, such as edema and erythema, appeared ten days after primary

5 immunization. The vehicle control group reached maximum paw swelling 19 days after the

6 first immunization, at which point it gradually diminished (Figure 1A). The two hind paws

7 were not significantly different from each other.

The effects of different total saponin doses from Achyranthes on the symptoms of CIA were the examined. Our results indicated that medium-dose $(150 \mathrm{mg} / \mathrm{kg})$ and high-dose (300 $\mathrm{mg} / \mathrm{kg}$ ) of total saponins resulted in a marked reduction on hind paw volume of arthritic rats

11 when compared to vehicle controls $(* P<0.05, * * P<0.01)$, however, Low dose $(75 \mathrm{mg} / \mathrm{kg})$ showed no significant change compared to vehicle controls. Compared to healthy rats, the

13 subjects' overall weight in both vehicle and experimental groups was significantly reduced

14 (Figure 1B). Notably, the DEX treatment group subjects had paw volumes that continued to 15 decline, even past vehicle control. The serious emaciation of DEX group indicated critical 16 damage of organism that showed superiority of TWH and Achyranthes saponins in keeping 17 weight of rats. 


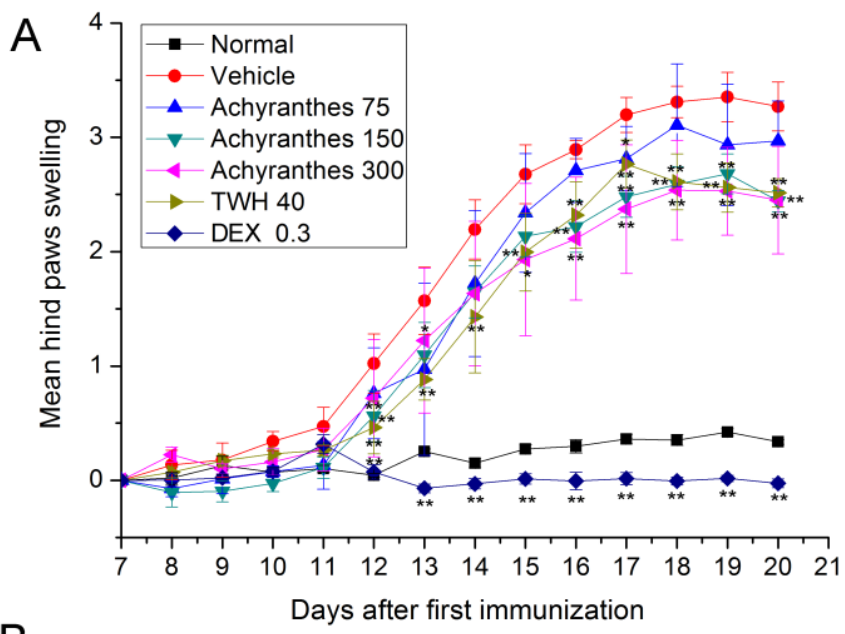

B

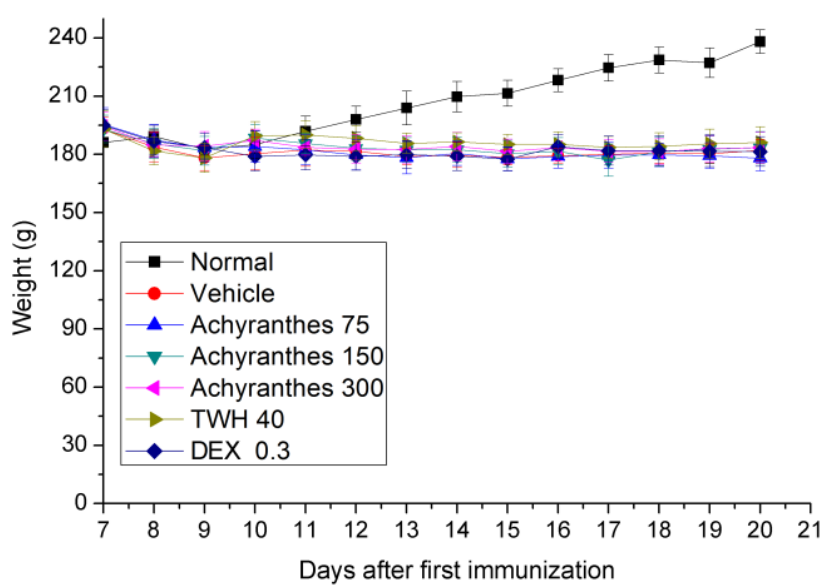

Figure 1. Measurement of mean hind paws swelling and weight. (A) Mean hind paws swelling changed seven days after the first immunization. Data are expressed as mean \pm SD $(n=10) . * P<0.05$; ** $\mathrm{P}<0.01$ vs. the vehicle arthritic rats. (B) Weight changes at seven days after first immunization.

Radiographs were taken to exam the bone degradation in the hind paws of Achyranthes saponins-treated arthritic rats. Representative hind paw photos and their corresponding

4 radiographs from normal, vehicle, and experimental rats are shown in Figure 2. These

5 representative photos show the characteristic severe soft tissue swelling and corresponding

6 radiographs revealbone erosion in the joints of the vehicle arthritic rats when compared to

7 healthy controls. Importantly, a high dose of Achyranthes saponins $(300 \mathrm{mg} / \mathrm{kg}$ ) was found to 
markedly inhibit the soft tissue swelling and bone erosion of arthritic rat joints.

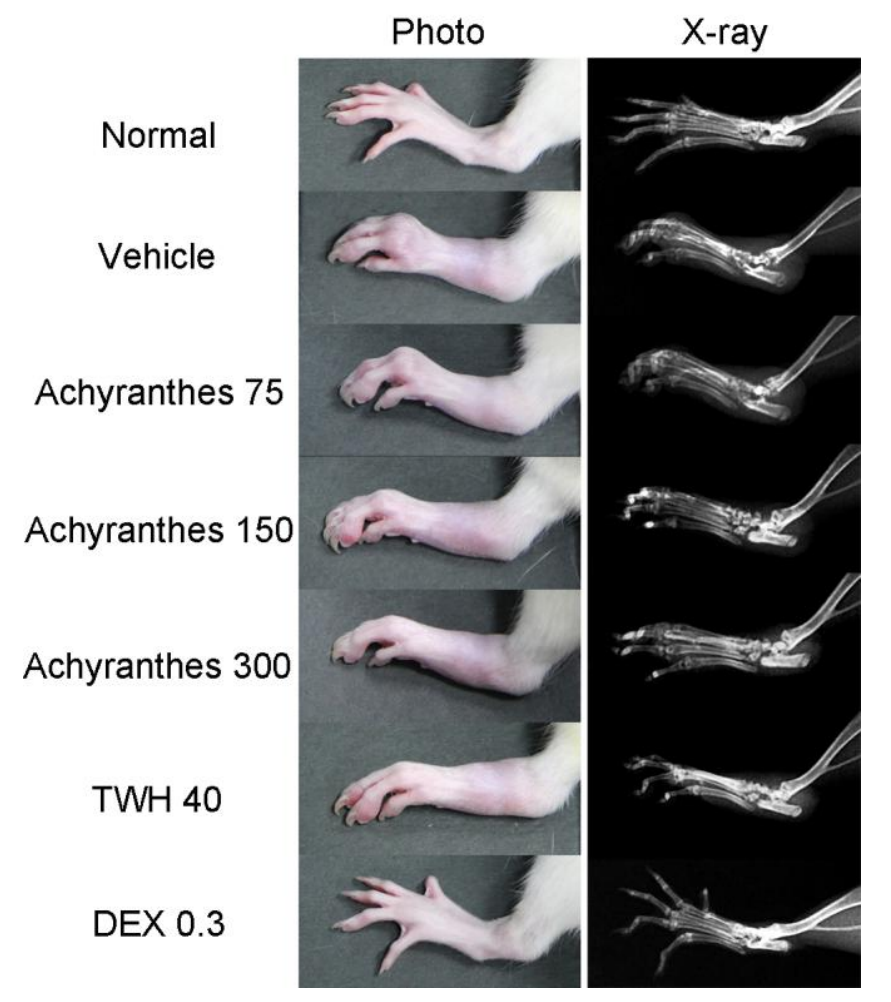

Figure 2. Representative photos and their corresponding radiographs of the hind paws from normal, vehicle, and drug-treated rats at twenty days post-initial immunization. Rats received a daily, oral treatment that contained different doses of Achyranthes saponins, TWH, DEX, or vehicle. These treatments began on day 8 after the first immunization. The left hind paw of each group was used for photographs and radiographs.

A histopathological examination was performed to detect joint inflammation in the hind

4 paws of Achyranthes saponins-treated arthritic rats. Representative histological sections of the ankle joints from normal, vehicle, and experimental rats are shown in Figure 3. The normal 6 rat joint presented with sound structural integrity along with a clean joint space and a smooth 7 articular cartilage surface. There were no detectable indicators of either synovial cell 8 proliferation or synovial tissue edema (Figure 3A, B and C). In contrast, the synovial joint 
1 space of vehicle rat was markedly narrower than normal subjects (Figure 3A). Furthermore,

2 the synovial joint showed varying degrees synovial hyperplasia (marked by black star in

3 Figure 3B), fiber tissue hyperplasia (shown as fibroblast proliferation in Figure 3B and C),

4 and inflammatory cellular infiltration (e.g. neutrophil and monocyte in Figure 3B and C) in

$5 \quad$ vehicle control and other drug treated groups. Pyocyte were observed in the joints of vehicle

6 control, Achyranthes 75 and 150 rats which presented severe inflammation. Finally, the joints

7 of vehicle control had obvious cartilage erosion and bone hyperplasia destruction (Figure 3A).

8 Notably, rats treated with high doses of Achyranthes saponins $(300 \mathrm{mg} / \mathrm{kg})$ had less joint

9 inflammation and synovial hyperplasia. 


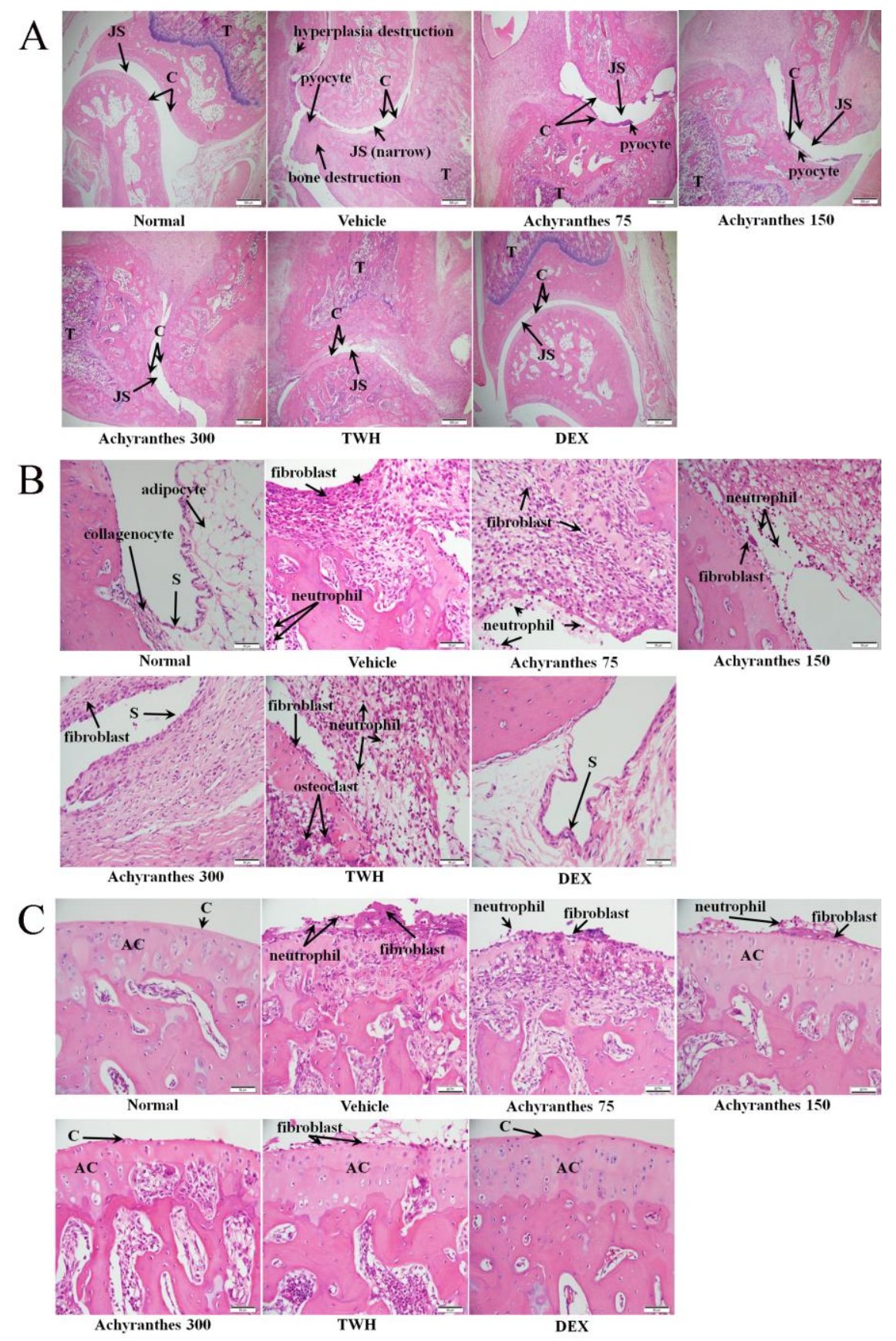

Figure 3. Histopathological changes of rat joints from normal, vehicle, and drug-treated groups twenty days after the first immunization. Rats received a daily, oral treatment with different doses of saponins, TWH, DEX, or vehicle, beginning on day 8 after the first immunization. The left hind paw of each group was taken to produce the histological sections. (A) The representative H\&E stained histopathological sections of the ankle joints from normal, vehicle, three doses of Achyranthes saponins, TWH, and DEX arthritic rats are shown (original magnification $\times 40$ ). The scaleplate 
represents $500 \mu \mathrm{m}$. (B) Representative H\&E stained histopathological sections of joint capsule from normal, vehicle, three doses of Achyranthes saponins, TWH, and DEX arthritic rats are shown (original magnification $\times 400$ ). The scaleplate represents $50 \mu \mathrm{m}$. (C) The representative H\&E stained histopathological sections of ankle joint articular surface from normal, vehicle, three doses of Achyranthes saponins, TWH, and DEX arthritic rats are shown (original magnification $\times 400$ ). The scaleplate represents $50 \mu \mathrm{m}$. Key: AC, articular cartilage; C, cartilage; JS, joint space; S, synovial; T, tibia.

2D-DIGE was used to understand both the molecular pathogenesis of RA and identify possibly biomarkers resulting from Achyranthes saponins therapy. Normal, vehicle, and the high dose of Achyranthes $(300 \mathrm{mg} / \mathrm{kg}$ ) groups were chosen for the proteomics study as this dose showed the most anti-arthritic effect. All biological replicates DIGE images were displayed in Supplementary Figure S3. Twenty-eight spots were selected that significantly

8 differential expressed $(P<0.05)$ between normal and vehicle groups, between vehicle and

9 the Achyranthes $(300 \mathrm{mg} / \mathrm{kg})$ groups, or between the Achyranthes $(300 \mathrm{mg} / \mathrm{kg})$ groups and normal (Figure 4). Among the differentially expressed spots, 23 proteins were successfully

11 identified by MALDI-TOF/TOF MS. The qualitative analysis showed that 16 proteins were 12 significantly increased and six significantly decreased when comparing vehicle to controls.

13 Seven proteins were significantly increased and 14 significantly decreased in Achyranthes experimental group when compared to vehicle. Additionally, 11 proteins were significantly increased and 7 significantly decreased in Achyranthes experimental group when compared to 

proteome annotation (Supplementary Figure S4).

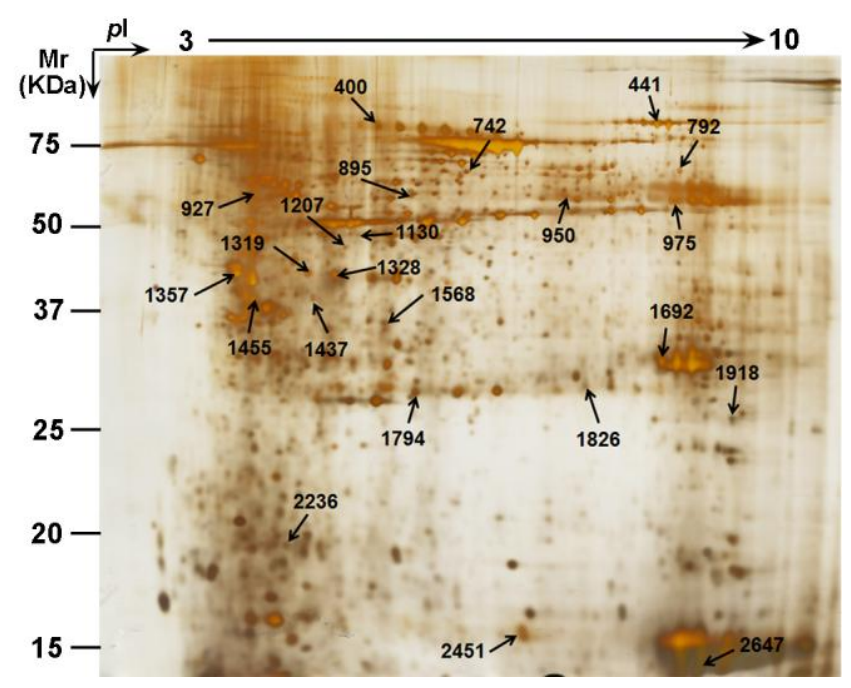

Figure 4. The differential protein spots identified by MALDI-TOF/TOF MS. Twenty-five differentially expressed protein spots were marked by their respective number and arrows on the silver stained gel.

53.3 Immunoblotting Analysis of RA Pathogenesis and Achyranthes Treatment-Related Proteins Immunoblot analysis was used to confirm the expression patterns of two candidate (PDIA3) and vimentin, which were changed significantly in vehicle group and recovered to nornal group after drug treatment (Figure 5A). Our statistical analysis on relative expression

11 indicated that both PDIA3 and vimentin were significantly increased in vehicle rats as compared to normal rats. Importantly, these two proteins decreased in rats receiving 

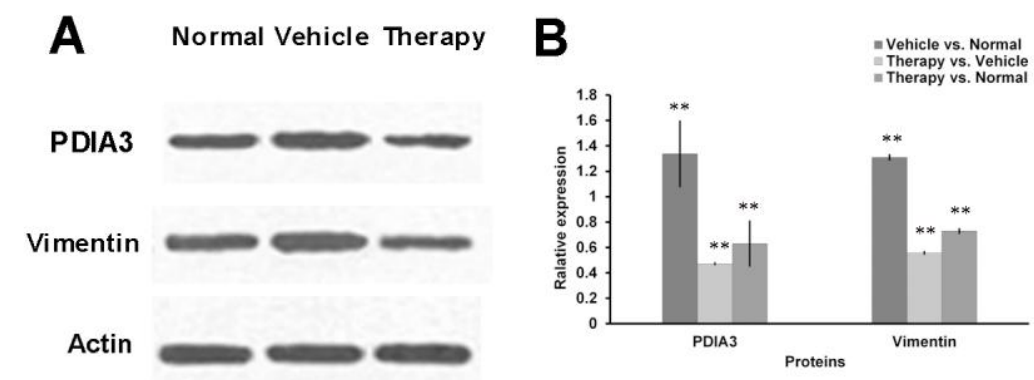

Figure 5. Immunoblotting analysis of selected candidate biomarkers for RA pathological process and therapy targets. (A) Representative Western blot of vimentin and protein disulfide-isomerase A3 (PDIA3) in normal, vehicle, and Achyranthes-treated groups. (B) Relative expression (mean \pm SD) of vimentin and protein disulfide-isomerase A3 (PDIA3) in normal, vehicle, and Achyranthes-treated groups. The actin was used to normalize (** $P<$ $0.01)$.

\section{Discussion}

A. aspera is one of the ethnomedicines used by the She ethnic minority in China. Its first known record is in "Draft of Materia Medica" (Chinese, "Ben cao tu jing") during the Song Dynasty of China. As a member of TCMs, it is used in combination with Himalayan teasel root and chaenomeles to treat rheumatoid arthritis. These effects have been recapitulated in rodent models of RA. For instance, extracts from $95 \%$ ethanol of A. aspera inhibited carrageenan-induced paw edema in a model of Freund's complete adjuvant-induced arthritis [12]. Quercetin and rhamnoglucoside were considered contributing factors to inhibit the edema produced by carrageenan [13]. Prior work has shown that a triterpene saponin isolated from TCMs application leads to pro-inflammatory cytokine and mediator regulation of RA in rats. Moreover, that TNF- $\alpha$ and COX-2 may be the effective targets in the serum and synovial 
membrane of CIA rats [24]. A second saponin has also been shown to alter serum levels in

CIA-treated mice of anti-CII IgG2a antibody, prostaglandin E2, TNF- $\alpha$, IL-1 $\beta$, and IL-6 [25]. However, there has been no work done on the details of what molecular mechanism is responsible for the anti-inflammation and anti-arthritic activities of these saponins. In this study, the total sapnonins extracted from A. aspera were shown to relieve RA symptoms in CIA rats, including ankle joint edema (Figure 2), synovial cell hyperplasia (Figure 3B), inflammatory cells infiltration (Fiugre 3B and C), and injury of articular cartilage (Figure 3A and C). These results indicate that A. aspera is a promising candidate for arthritic therapy. Moreover, that saponin is the effective compound that possesses the anti-inflammation and anti-arthritic activities that have been previously reported.

Our histopathological result showed obvious fibroblast hyperplasia and inflammatory cellular infiltration (e.g. neutrophil and monocyte) in joint capsule and articular surface afflicted with RA (Figure 3). Correspondingly, our proteomics result showed that fibrinogen B beta chain and the fibrinogen gamma chain were detected at higher levels in vehicle-treated as compared to normal rats (2.43 and 1.73 times higher, respectively, $P<0.05)$. Moreover, these two proteins decreased 3.95 and 1.71 times, respectively, in the Achyranthes-treated when compared to vehicle-treated rats $(P<0.05)$ (Table 1). Fibrinogen has been postulated to be a potent RA inducer and plasminogen has been reported to have opposing effects on paw and knee joints in TNF- $\alpha$-driven arthritic mice. Importantly, both the exacerbated arthritic severity in the paws joints and the dramatically decreased arthritic symptoms within the knee joints were mechanistically linked to fibrinogen [26]. This past work is further supported by our fibrinogen expression results from the synovial tissue of CIA rats. Furthermore, we 
speculate that the fibrinogen B beta chain is more sensitive to RA-derived inflammation and

2 also to subsequent saponin therapy.

The procollagen type I and type VI, apolipoprotein A-I (apoA-I), and PDIA3 had the same expression pattern as fibrinogen in that they all had significantly increased levels in synovial tissues of vehicle CIA rats. Additionally, they all had significantly decreased levels in the synovial tissue derived from drug-treated rats. The post-translationally modified (citrullinated) fibrinogen is highly capable of inducing arthritis. Prior work has shown that there is IgG variability in response to particular fibrinogen-derived antigens from individual mice [27]. Fibrinogen, collagen, and vimentin can induce anti-cyclic, citrullinated peptide antibodies as autoantigens by rheumatoid factors [28]. Metalloproteinases, an interstitial collagenase, plays a key part in the destruction of joints in rheumatoid arthritis and initiates the degradation of type I and III collagens [29, 30]. The collagen type I alpha 1 increased over two times in the RA synovium of rats (Table 1) implies metalloproteinase inhibition and less destruction of joints in rheumatoid arthritis. It has been reported that apoA-I was up-regulated in infectious bursal disease in virus-infected chicken embryo fibroblasts [31]. Furthermore, that apo A-I was heavily secreted from chondrocytes in subjects' knees that had received surgical depositions of amyloid [32]. Apo A-I has also been considered a valid biomarker for the infliximab response to rheumatoid arthritis [33]. In this study, the promotion of apo A-I in inflamed synovial tissue was presumed to be an autoimmune mechanism in the monocyte-macrophages that had been activated by $\mathrm{T}$ lymphocytes. This caused an inhibition of both TNF- $\alpha$ and IL-1 $\beta$ production [34].

Protein disulfide isomerase is a key enzyme that catalyzes the disulfide formation and 
1 isomerization in compact folding intermediates [35]. PDIA3 (also called thiol-oxidoreductase

2 ERp57, ER60, or GRP58) is one member of the protein disulfide isomerase superfamily and

3 is essential for the stable assembly of the peptide-loading complex and its export from

4 the endoplasmic reticulum to the cell surface [36]. PDIA3, superoxide dismutase (SOD), and

5 procollagen were robustly up-regulated in chondrocyte-like cells during chondrogenesis in vitro [37]. According to our 2D DIGE results, both PDIA3 and SOD were both elevated more

7 than 1.5 times in the inflamed synovial tissue compared to normal. Our immunoblotting

8 results further confirmed the significant difference in expression of PDIA3 between

9 vehicle-treated and normal rats. Collectively, these results are in accordance with the histopathology of articular cartilage proliferation in the progression of arthritis. Immune complexes of IgG are mostly in plasma and, less frequently, in synovial fluid. They are often pathogenic and work by activating complement and stimulating cytokine 13 synthesis [38]. In the synovial tissue, IgG-2a was detected at levels 1.7-fold higher in inflamed synovial tissue compared to normal. It is a special autoantigen that did not have any significant change during saponin therapy, thus indicating it as a negative therapy target of Achyranthes saponin. The alpha-enolase, a glycolytic enzyme expressed in most tissues, had the most similar expression pattern when compared to IgG. Its increasing expression has been reported in monocytes and macrophages isolated from the synovium of RA patients and the arthritic ankle tissues of mice with collagen-induced arthritis [39]. These past findings are consistent with our current results.

Vimentin, a major cytoskeleton component, is detectable in synovium, fibroblast-like synoviocytes, and vascular endothelial lining cells [40,41]. Vimentin was reported as being 
1 highly phosphorylated in tumors that do not overexpress epidermal growth factor receptor

2 tyrosine kinase and that it is associated with tumor growth, invasion, drug resistance, and poor

3 prognosis [42]. As confirmed by western blotting, the vimentin could have positive

4 correlation with inflammation invasion degree and sensitive to Achyranthes saponins

5 treatment in the synovium of CIA rats.

Alpha-1-antiproteinase (also known as alpha 1-antitrypsin, $\alpha \mathrm{AT}$ ), is a serpins superfamily member and is considered inhibit a wide variety of proteases [43]. The secretion of $\alpha \mathrm{AT}$ has been shown to be associated with most cancer and inflammatory disease in vitro and in vivo. Increasing levels of $\alpha \mathrm{AT}$ in drug-treated rats may be associated with the inhibition of inflammation and a negative correlation of the TNF-a concentration to levels of $\alpha 1 \mathrm{AT}$ inactivation [44]. The manganese superoxide dismutase (MnSOD) is a mitochondrial matrix protein that serves a protective function from reactive oxygen species. Over-expression of extracellular superoxide dismutase in mouse synovial tissue contributed to the attenuation of the inflammation and inhibition of IL- $1 \beta, \mathrm{TNF}-\alpha$, and matrix metalloproteinase in fibroblast-like synoviocytes [45]. Moreover, MnSOD was found to decrease in osteoarthritic chondrocytes and as a key factor in redox imbalance in osteoarthritis [46]. However, there have been conflicting reports stating that MnSOD was induced by the chondroitin sulfate treatment in osteoarthritis [47]. In our current results, MnSOD increased after Achyranthes saponins treatment which was in line with the latter findings. It implied the protective function of MnSOD from reactive oxygen species could benefit for RA therapy.

In the current study, Achyranthes saponins have been shown to be the active component in slowing the progression of joint swelling, soft tissue inflammation, and bone erosion in 
1 orally-treated, CIA rats. These results indicate that Achyranthes aspera is a potential

2 candidate for future clinical trials on RA patients. Alterations in fibrinogen, procollagen, and

3 apoA-I levels indicate pathology and mechanistic association with RA-induced inflammation.

4 Vimentin and PDIA3 could be the candidate biomarkers specific to Achyranthes saponins 5 therapy for rheumatoid arthritis in synovial membrane.

6

\section{Acknowledgments}

8 This work was supported by the Zhejiang Provincial Natural Science Foundation of China

9 (Grant No. Y2100765), the National Science Foundation of China (Grant No. U1303122),

10 Zhejiang Province Science and Technology Innovation team (Grant No. 201210044-10). 


\section{Reference}

[1] P.C. Taylor, M. Feldmann, Anti-TNF biologic agents: still the therapy of choice for rheumatoid arthritis, Nature Reviews Rheumatology, 5 (2009) 578-582.

[2] V. Ramgolam, S. Ang, Y. Lai, C. Loh, H. Yap, Traditional Chinese medicines as immunosuppressive agents, Ann. Acad. Med. Singapore, 29 (2000) 11-16.

[3] H. Aikman, The association between arthritis and the weather, IJBm, 40 (1997) 192-199.

[4] A.A. Gorin, J.M. Smyth, J.N. Weisberg, G. Affleck, H. Tennen, S. Urrows, A.A. Stone, Rheumatoid arthritis patients show weather sensitivity in daily life, but the relationship is not clinically significant, Pain, 81 (1999) 173-177.

[5] A. Chakraborty, A. Brantner, T. Mukainaka, Y. Nobukuni, M. Kuchide, T. Konoshima, H. Tokuda, H. Nishino, Cancer chemopreventive activity of Achyranthes aspera leaves on Epstein-Barr virus activation and two-stage mouse skin carcinogenesis, Cancer Lett., 177 (2002) 1-5.

[6] R. Chakrabarti, P.K. Srivastava, K. Kundu, R.S. Khare, S. Banerjee, Evaluation of immunostimulatory and growth promoting effect of seed fractions of Achyranthes aspera in common carp Cyprinus carpio and identification of active constituents, Fish Shellfish Immunol., 32 (2012) 839-843.

[7] A. Fikru, E. Makonnen, T. Eguale, A. Debella, G. Abie Mekonnen, Evaluation of in vivo wound healing activity of methanol extract of Achyranthes aspera L, J. Ethnopharmacol., 143 (2012) 469-474. [8] S. Srivastav, P. Singh, G. Mishra, K. Jha, R. Khosa, Achyranthes aspera-An important medicinal plant: A review, J Nat Prod Plant Resour, 1 (2011) 1-14.

[9] A. Gomes, R. Das, S. Sarkhel, R. Mishra, S. Mukherjee, S. Bhattacharya, A. Gomes, Herbs and herbal constituents active against snake bite, Indian J. Exp. Biol., 48 (2010) 865-878. 
[10] A. Fikru, E. Makonnen, T. Eguale, A. Debella, G. Abie Mekonnen, Evaluation of $<$ i $>$ in vivo $</$ i $>$ wound healing activity of methanol extract of $<\mathrm{i}>$ Achyranthes aspera L</i $>$, J. Ethnopharmacol., 143 (2012) 469-474.

[11] F. Khuda, Z. Iqbal, A. Khan, Y. Shah, L. Ahmad, F. Nasir, M. Hassan, W.A. Shah, Evaluation of anti-inflammatory activity of selected medicinal plants of Khyber Pakhtunkhwa, Pakistan, Pak. J. Pharm. Sci., 27 (2014) 365-368.

[12] A.B. Gokhale, A.S. Damre, K.R. Kulkami, M.N. Saraf, Preliminary evaluation of anti-inflammatory and anti-arthritic activity of S. lappa, A. speciosa and A. aspera, Phytomedicine, 9 (2002) 433-437.

[13] T. Vetrichelvan, M. Jegadeesan, Effect of alcohol extract of Achyranthes aspera Linn. on acute and subacute inflammation, Phytother. Res., 17 (2003) 77-79.

[14] S. Hanash, Disease proteomics, Natur, 422 (2003) 226-232.

[15] K. Tilleman, K. Van Beneden, A. Dhondt, I. Hoffman, F. De Keyser, E. Veys, D. Elewaut, D. Deforce, Chronically inflamed synovium from spondyloarthropathy and rheumatoid arthritis investigated by protein expression profiling followed by tandem mass spectrometry, Proteomics, 5 (2005) 2247-2257.

[16] A. Sinz, M. Bantscheff, S. Mikkat, B. Ringel, S. Drynda, J. Kekow, H.J. Thiesen, M.O. Glocker, Mass spectrometric proteome analyses of synovial fluids and plasmas from patients suffering from rheumatoid arthritis and comparison to reactive arthritis or osteoarthritis, Electrophoresis, 23 (2002) 3445-3456.

[17] T. Uchida, A. Fukawa, M. Uchida, K. Fujita, K. Saito, Application of a novel protein biochip technology for detection and identification of rheumatoid arthritis biomarkers in synovial fluid, J. 
Proteome Res., 1 (2002) 495-499.

[18] K. Dasuri, M. Antonovici, K. Chen, K. Wong, K. Standing, W. Ens, H. El-Gabalawy, J.A. Wilkins, The synovial proteome: analysis of fibroblast-like synoviocytes, Arthritis Res. Ther., 6 (2004) R161.

[19] H. Dotzlaw, M. Schulz, M. Eggert, G. Neeck, A pattern of protein expression in peripheral blood mononuclear cells distinguishes rheumatoid arthritis patients from healthy individuals, Biochim. Biophys. Acta, 1696 (2004) 121-129.

[20] P. Lorenz, M. Bantscheff, S.M. Ibrahim, H.J. Thiesen, M.O. Glocker, Proteome analysis of diseased joints from mice suffering from collagen-induced arthritis, Clin. Chem. Lab. Med., 41 (2003) $1622-1632$.

[21] A. Shevchenko, M. Wilm, O. Vorm, M. Mann, Mass spectrometric sequencing of proteins from silver-stained polyacrylamide gels, Anal. Chem., 68 (1996) 850-858.

[22] J. Li, H. Qi, L.-W. Qi, L. Yi, P. Li, Simultaneous determination of main phytoecdysones and triterpenoids in Radix Achyranthis Bidentatae by high-performance liquid chromatography with diode array-evaporative light scattering detectors and mass spectrometry, Anal. Chim. Acta, 596 (2007)

264-272.

[23] G. Michl, D. Abebe, F. Bucar, A. Debella, O. Kunert, M.G. Schmid, E. Mulatu, E. Haslinger, New Triterpenoid Saponins from Achyrantes asperaLinn, AcHCh, 83 (2000) 359-363.

[24] C. Peng, P.K. Perera, Y.M. Li, W.R. Fang, L.F. Liu, F.W. Li, Anti-inflammatory effects of Clematis chinensis Osbeck extract(AR-6) may be associated with NF-kappaB, TNF-alpha, and COX-2 in collagen-induced arthritis in rat, Rheumatol. Int., 32 (2012) 3119-3125.

[25] H.W. Jung, J.K. Jung, K.H. Son, D.H. Lee, T.M. Kang, Y.S. Kim, Y.K. Park, Inhibitory effects of the root extract of Dipsacus asperoides C.Y. Cheng et al T.M.Ai on collagen-induced arthritis in mice, 
J. Ethnopharmacol., 139 (2012) 98-103.

[26] H. Raghu, M. J Flick, Targeting the coagulation factor fibrinogen for arthritis therapy, Curr.

Pharm. Biotechnol., 12 (2011) 1497-1506.

[27] J.A. Hill, D.A. Bell, W. Brintnell, D. Yue, B. Wehrli, A.M. Jevnikar, D.M. Lee, W. Hueber, W.H.

Robinson, E. Cairns, Arthritis induced by posttranslationally modified (citrullinated) fibrinogen in

DR4-IE transgenic mice, J. Exp. Med., 205 (2008) 967-979.

[28] R.J. Seward, E.E. Drouin, A.C. Steere, C.E. Costello, Peptides presented by HLA-DR molecules in synovia of patients with rheumatoid arthritis or antibiotic-refractory Lyme arthritis, Mol. Cell . Proteomics, 10 (2011) M110 002477.

[29] Y. Okada, N. Takeuchi, K. Tomita, I. Nakanishi, H. Nagase, Immunolocalization of matrix metalloproteinase 3 (stromelysin) in rheumatoid synovioblasts (B cells): correlation with rheumatoid arthritis, Ann. Rheum. Dis., 48 (1989) 645-653.

[30] B. Pilcher, B. Sudbeck, J. Dumin, H. Welgus, W. Parks, Collagenase-1 and collagen in epidermal repair, Archives of dermatological research, 290 (1998) S37-S46.

[31] X. Zheng, L. Hong, L. Shi, J. Guo, Z. Sun, J. Zhou, Proteomics analysis of host cells infected with infectious bursal disease virus, Mol. Cell. Proteomics, 7 (2008) 612-625.

[32] A. Solomon, C.L. Murphy, D. Kestler, D. Coriu, D.T. Weiss, J. Makovitzky, P. Westermark, Amyloid contained in the knee joint meniscus is formed from apolipoprotein A-I, Arthritis Rheum., 54 (2006) 3545-3550.

[33] C. Trocme, H. Marotte, A. Baillet, B. Pallot-Prades, J. Garin, L. Grange, P. Miossec, J. Tebib, F. Berger, M.J. Nissen, R. Juvin, F. Morel, P. Gaudin, Apolipoprotein A-I and platelet factor 4 are biomarkers for infliximab response in rheumatoid arthritis, Ann. Rheum. Dis., 68 (2009) 1328-1333. 
[34] D. Burger, J.-M. Dayer, High-density lipoprotein-associated apolipoprotein AI: the missing link between infection and chronic inflammation?, Autoimmun. Rev., 1 (2002) 111-117.

[35] M. Molinari, A. Helenius, Glycoproteins form mixed disulphides with oxidoreductases during folding in living cells, Natur, 402 (1999) 90-93.

[36] N. Garbi, S. Tanaka, F. Momburg, G.J. Hammerling, Impaired assembly of the major histocompatibility complex class I peptide-loading complex in mice deficient in the oxidoreductase ERp57, Nat. Immunol., 7 (2006) 93-102.

[37] A. De la Fuente, J. Mateos, I. Lesende-Rodriguez, V. Calamia, I. Fuentes-Boquete, F.J. de Toro, M.C. Arufe, F.J. Blanco, Proteome analysis during chondrocyte differentiation in a new chondrogenesis model using human umbilical cord stroma mesenchymal stem cells, Mol. Cell . Proteomics, 11 (2012) M111 010496.

[38] M. Feldmann, F.M. Brennan, R.N. Maini, Rheumatoid arthritis, Cell, 85 (1996) 307-310.

[39] S. Bae, H. Kim, N. Lee, C. Won, H.R. Kim, Y.I. Hwang, Y.W. Song, J.S. Kang, W.J. Lee, alpha-Enolase expressed on the surfaces of monocytes and macrophages induces robust synovial inflammation in rheumatoid arthritis, J Immunol, 189 (2012) 365-372.

[40] F. Ota, A. Maeshima, S. Yamashita, H. Ikeuchi, Y. Kaneko, T. Kuroiwa, K. Hiromura, K. Ueki, I. Kojima, Y. Nojima, Activin A induces cell proliferation of fibroblast - like synoviocytes in rheumatoid arthritis, Arthritis Rheum., 48 (2003) 2442-2449.

[41] O.A. Osung, M. Chandra, E.J. Holborow, Intermediate filaments in synovial lining cells in rheumatoid arthritis and other arthritides are of vimentin type, Ann. Rheum. Dis., 41 (1982) 74-77. [42] H. Johnson, A.M. Del Rosario, B.D. Bryson, M.A. Schroeder, J.N. Sarkaria, F.M. White, Molecular characterization of EGFR and EGFRvIII signaling networks in human glioblastoma tumor 
xenografts, Mol. Cell. Proteomics, 11 (2012) 1724-1740.

[43] P.G. Gettins, Serpin structure, mechanism, and function, Chem. Rev., 102 (2002) 4751-4804.

[44] K. Chidwick, C.E. Whichelow, Z. Zhang, K. Fairburn, J.A. Sachs, D.R. Blake, P.G. Winyard, Relationship between alpha 1-antitrypsin inactivation and tumor necrosis factor alpha concentration in the synovial fluid of patients with rheumatoid arthritis, Arthritis Rheum., 37 (1994) 1723-1726.

[45] D.H. Yu, J.K. Yi, H.S. Yuh, S. Park, H.J. Kim, K.B. Bae, Y.R. Ji, N.R. Kim, S.J. Park, H. Kim do, S.H. Kim, M.O. Kim, J.W. Lee, Z.Y. Ryoo, Over-expression of extracellular superoxide dismutase in mouse synovial tissue attenuates the inflammatory arthritis, Exp. Mol. Med., 44 (2012) 529-535.

[46] C. Ruiz-Romero, V. Calamia, J. Mateos, V. Carreira, M. Martinez-Gomariz, M. Fernandez, F.J. Blanco, Mitochondrial dysregulation of osteoarthritic human articular chondrocytes analyzed by proteomics: a decrease in mitochondrial superoxide dismutase points to a redox imbalance, Mol. Cell . Proteomics, 8 (2009) 172-189.

[47] V. Calamia, P. Fernandez-Puente, J. Mateos, L. Lourido, B. Rocha, E. Montell, J. Verges, C. Ruiz-Romero, F.J. Blanco, Pharmacoproteomic study of three different chondroitin sulfate compounds on intracellular and extracellular human chondrocyte proteomes, Mol. Cell . Proteomics, 11 (2012) M111 013417. 
Table 1. Differential proteins in synovium of different groups of rats identified by 2D-DIGE proteomics and MALDI-TOF/TOF mass spectrometry.

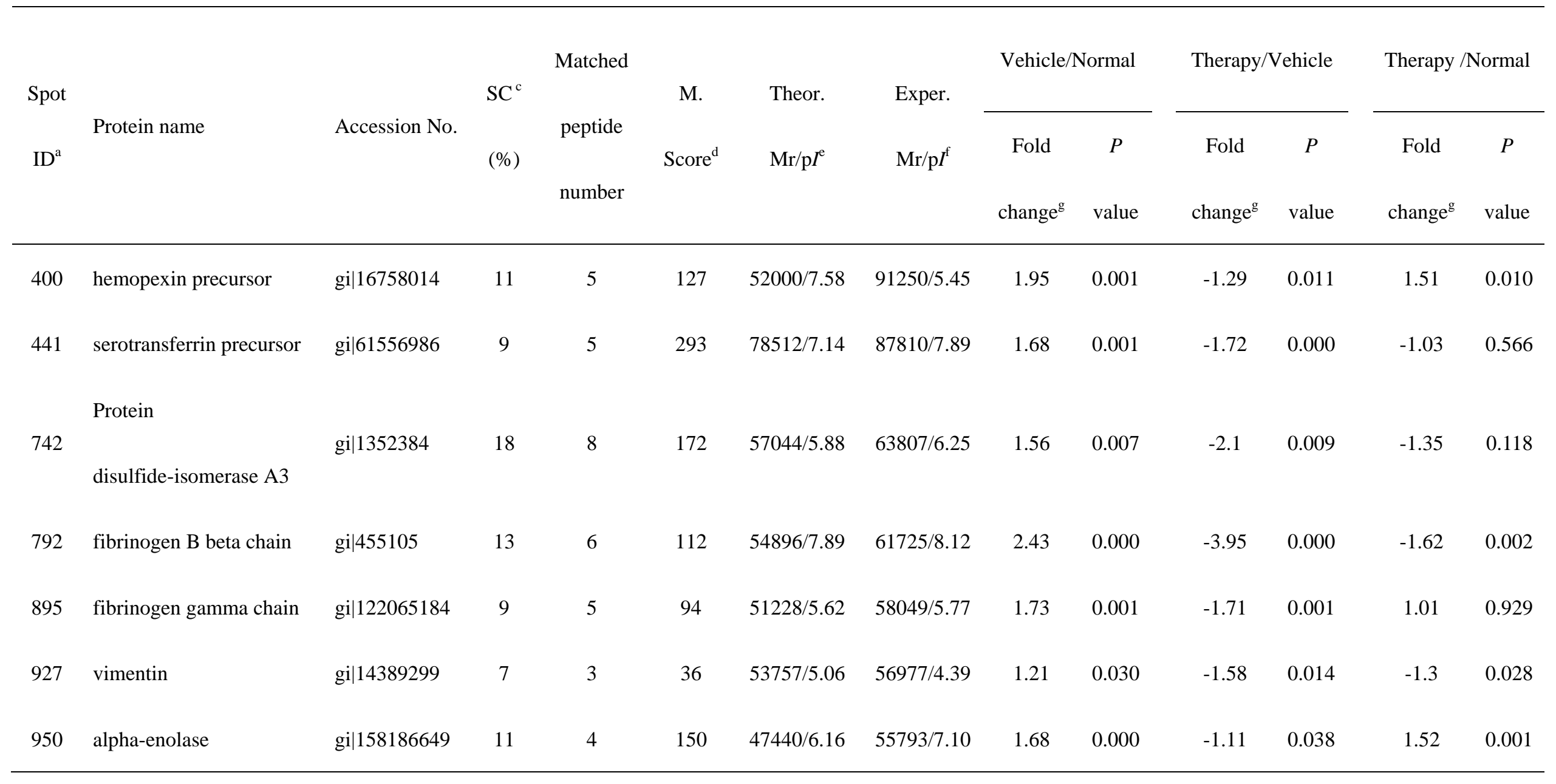




\begin{tabular}{|c|c|c|c|c|c|c|c|c|c|c|c|c|c|}
\hline 975 & IgG-2a protein & gi|1220486 & 6 & 3 & 40 & $50977 / 7.52$ & $54833 / 7.20$ & 1.71 & 0.000 & 1 & 0.995 & 1.71 & 0.000 \\
\hline 1130 & $\operatorname{lamin} \mathrm{A}$ & gi| 453180 & 8 & 5 & 81 & $71884 / 6.20$ & $49327 / 5.43$ & -1.68 & 0.001 & -1.01 & 0.934 & -1.69 & 0.001 \\
\hline 1207 & $\begin{array}{l}\text { procollagen, type VI, } \\
\text { alpha 3, isoform CRA_a }\end{array}$ & gi|149037629 & 3 & 5 & 53 & $240932 / 5.26$ & $46914 / 5.16$ & 1.07 & 0.037 & -1.96 & 0.001 & -1.84 & 0.001 \\
\hline 1319 & haptoglobin precursor & gi|60097941 & 14 & 5 & 84 & $39052 / 6.10$ & $42765 / 4.82$ & 1.66 & 0.000 & -1.32 & 0.003 & 1.26 & 0.000 \\
\hline 1328 & haptoglobin precursor & gi|60097941 & 9 & 5 & 69 & $39052 / 6.10$ & $42566 / 5.08$ & 1.65 & 0.000 & -1.17 & 0.035 & 1.41 & 0.000 \\
\hline 1357 & $\begin{array}{l}\text { smooth-muscle alpha } \\
\text { tropomyosin }\end{array}$ & gi|207350 & 22 & 6 & 288 & $32713 / 4.74$ & $41623 / 4.17$ & -1.69 & 0.002 & -1.55 & 0.046 & -2.62 & 0.000 \\
\hline 1437 & $\begin{array}{l}\text { procollagen, type } 1 \text {, alpha } \\
\text { 1, isoform CRA_a }\end{array}$ & gi|149053909 & 3 & 2 & 45 & $72112 / 5.95$ & $38853 / 4.87$ & 2.15 & 0.000 & -1.54 & 0.001 & 1.39 & 0.003 \\
\hline 1455 & vimentin & gi|14389299 & 18 & 7 & 205 & $53757 / 5.06$ & $38430 / 4.38$ & 1.60 & 0.003 & -1.69 & 0.018 & -1.69 & 0.775 \\
\hline 1568 & Alpha-1-antiproteinase & gi|112889 & 8 & 3 & 51 & $46278 / 5.70$ & $35102 / 5.56$ & 1.25 & 0.045 & 2.45 & 0.002 & 3.07 & 0.002 \\
\hline 1692 & carbonic anhydrase 3 & gi|31377484 & 31 & 6 & 209 & $29698 / 6.89$ & $32155 / 8.02$ & -1.93 & 0.001 & 1.47 & 0.002 & -1.31 & 0.014 \\
\hline
\end{tabular}




\begin{tabular}{|c|c|c|c|c|c|c|c|c|c|c|c|c|c|}
\hline 1794 & apolipoprotein A-I & gi|2145143 & 30 & 6 & 258 & $29869 / 5.51$ & $30082 / 5.84$ & 2.21 & 0.000 & -1.74 & 0.000 & 1.27 & 0.003 \\
\hline 1826 & carbonic anhydrase III & gi|203225 & 13 & 4 & 76 & $29668 / 6.49$ & $29524 / 7.32$ & -1.81 & 0.003 & 2.84 & 0.002 & 1.57 & 0.011 \\
\hline & superoxide dismutase & 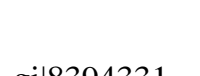 & 0 & 0 & 50 & $24007 / 006$ & $27121 / 0$ & 201 & 0040 & 176 & ת & 20 & $\rho \cap \cap 1$ \\
\hline \multirow{2}{*}{1918} & {$[\mathrm{Mn}]$} & & & & & & & & & & & & \\
\hline & mitochondrial & & & & & & & & & & & & \\
\hline \multirow[t]{3}{*}{2236} & synthase, $\mathrm{H}+$ transporting & gi|89574017 & 10 & 2 & 105 & $37228 / 4.97$ & $19717 / 4.22$ & -2.42 & 0.000 & 2.43 & 0.000 & 1.01 & 0.898 \\
\hline & F1 complex beta subunit & & & & & & & & & & & & \\
\hline & acid-binding & & & & & & & & & & & & \\
\hline \multirow[t]{2}{*}{2451} & & gi|2494405 & 26 & 3 & 96 & $14813 / 7.71$ & $16851 / 6.82$ & -2.17 & 0.002 & 1.34 & 0.006 & -1.62 & 0.004 \\
\hline & protein, adipocyte & & & & & & & & & & & & \\
\hline 2647 & hemoglobin, alpha 2 & gi|60678292 & 50 & 5 & 227 & $15446 / 8.45$ & $13353 / 8.39$ & 1.04 & 0.699 & 2.72 & 0.003 & 2.82 & 0.003 \\
\hline
\end{tabular}

${ }^{a}$ Spot ID was created by the Decyder2D software and marked in Figure 4.

${ }^{\mathrm{b}}$ Sequence coverage.

${ }^{c}$ Protein ID is the Mascot results of MALDI-TOF/TOF MS searched from the NCBInr database 
${ }^{\mathrm{d}}$ The Mascot score reported after searching against the NCBInr database. Protein scores were derived from ions scores as a non-probabilistic basis for ranking protein hits in the Mascot Search Results, individual ions scores $>43$ indicate identity or extensive homology $(P<0.05)$.

${ }^{\mathrm{e}}$ Theoretical mass $(\mathrm{kDa})$ and $\mathrm{pI}$ of identified proteins were retrieved from the protein database.

${ }^{\mathrm{f}}$ Experimental mass $(\mathrm{kDa})$ and $\mathrm{pI}$ of identified proteins. Experimental values were calculated with Image Master 2D Platinum Software using standard molecular mass markers.

${ }^{g}$ Average relative protein abundance change between two groups. Positive value indicates increase and negative value indicates decrease. 